\title{
Locally advanced non-small cell lung cancer: the place of specialist thoracic surgery in the multidisciplinary team
}

\author{
Muteb Al Zaidi ${ }^{1,2}$, Gavin M. Wright ${ }^{3,4,5}$ \\ ${ }^{1}$ Department of Cardiothoracic Surgery, St. Vincent's Hospital (Melbourne), University of Melbourne, Fitzroy, Victoria, Australia; ${ }^{2}$ Division of \\ Thoracic Surgery, King Abdullah Medical City, Makkah, Saudi Arabia; ${ }^{3}$ Department of Surgery, St. Vincent's Hospital (Melbourne), University of \\ Melbourne, Fitzroy, Victoria, Australia; ${ }^{4}$ Victorian Comprehensive Cancer Centre, Parkville, Victoria, Australia; ${ }^{5}$ Peter MacCallum Cancer Centre \\ and Royal Melbourne Hospital, Parkville, Victoria, Australia \\ Contributions: (I) Conception and design: GM Wright; (II) Administrative support: All authors; (III) Provision of study materials or patients: GM \\ Wright; (IV) Collection and assembly of data: All authors; (V) Data analysis and interpretation: All authors; (VI) Manuscript writing: All authors; (VII) \\ Final approval of manuscript: All authors. \\ Correspondence to: A/Professor Gavin M. Wright, MBBS (Melb), FRACS, PhD. 55 Victoria Parade, Fitzroy, Victoria 3065, Australia. \\ Email: Gavin.Wright@svha.org.au.
}

\begin{abstract}
One reason that lung cancer is the leading cause of cancer mortality worldwide, is that surgical intervention is highly dependent on earlier tumor stage and good patient condition. As large proportion of cases are already metastatic at presentation and many are locally advanced, curative surgery is only possible in a minority of fit patients. Increasing the number of patients achieving complete resection is one of the avenues to increase overall survival using our existing technology. In the past, complex cases may have been sporadically discussed between various specialists in order to achieve better outcomes. More recently, the idea of discussing those cases on a routine basis, rather than an accident of geography or referral pattern, gave rise to the multidisciplinary team. Lung cancer management is now increasingly complex, especially with novel modalities such as targeted therapies, immune checkpoint inhibitors and stereotactic body radiotherapy delivery. Likewise, in thoracic surgery, minimally invasive techniques, early recovery after surgery protocols and complex techniques for resecting locally advanced tumours or preserving lung parenchyma must all be deployed appropriately to continue our incremental gains in survival and quality of life. To highlight the role of specialist thoracic surgeon in the multidisciplinary care of locally advanced non-small cell lung cancer, we conducted a search of English language publications for its multidisciplinary-based surgical management. We limited our search to the last decade, then hand-searched relevant references. In addition, we used our large prospective database as a team-oriented specialized thoracic surgical service to benchmark and demonstrate the benefits of specialist surgeons in the modern multidisciplinary team. In conclusion, patients with locally advanced non-small cell lung cancer should have any surgical option withheld without a specialist thoracic surgical opinion as part of the multidisciplinary team discussion.
\end{abstract}

Keywords: Best practice analysis; interdisciplinary communication; pulmonary cancer; thoracic surgery; thoracic surgical procedures

Submitted Aug 03, 2019. Accepted for publication Nov 07, 2019.

doi: $10.21037 /$ tlcr.2019.11.22

View this article at: http://dx.doi.org/10.21037/tlcr.2019.11.22

\section{Introduction}

Traditionally, patients with locally advanced lung cancer were sporadically discussed in informal meetings of interested parties to achieve the best outcomes. Nowadays, the multidisciplinary meeting has an essential role not only in maximizing the survival of those patients (1-3), but to improve their quality of life $(4,5)$.

In order to achieve the aims of the modern 
multidisciplinary team (MDT), it is imperative that all relevant disciplines and specialties are involved in the patient's lung cancer management from diagnosis through to treatment and end of life care (4).

If not all patients, certainly those with locally advanced non-small cell lung cancer (LA-NSCLC), including superior sulcus tumors, lymph node involvement and those requiring neoadjuvant therapies, pneumonectomy or extended resections, should be discussed in an MDT composed of specialist thoracic surgeon, medical oncologist, radiation oncologist, respiratory physician, radiologist, pathologist, clinical nurse specialist, allied health disciplines with an MDT administrator. For these cases the MDT meeting not only ensures the best management plan can be offered, but maximizes the educational benefits of reviewing their diagnosis, staging and treatment plan.

Based on review evidence and the National Comprehensive Cancer Network (NCCN) guidelines 2019 (6), "thoracic surgeons should actively participate in multidisciplinary discussions and meetings regarding lung cancer patients". The NCCN guidelines further state: "Determination of resectability, surgical staging, and pulmonary resection should be performed by board-certified thoracic surgeons who perform lung cancer surgery as a prominent part of their practice." The strong message is that patients with LANSCLC should not be pronounced unresectable until specialist thoracic surgical opinion is provided. Therefore, the thoracic surgery team should have a major role in these discussions and ensure that all voices are heard. If resectable, but of high $\mathrm{T}$ stage or with mediastinal node involvement, then the decision needs to be made on whether this patient should be included in a protocol with or without surgery. Dedicated thoracic surgeons are adept at selecting patients that would do well with surgery, but realize that surgery alone is not a considered option. The local surgical experience may dictate whether radiation is entertained pre-operatively, as this may render the surgery more difficult or prone to post-operative complications. Whether a lung parenchyma-sparing operation, such as sleeve lobectomy or pulmonary arterial reconstruction, can avoid pneumonectomy is another decision to be made by the surgeon and this has an impact on adjuvant therapies.

To provide an evidence base for these tenets of thoracic oncology surgical practice, we conducted a review of literature on the role of surgery and the MDT meeting in LA-NSCLC for the last 10 years. We next queried our own extensive prospective databases as an experienced tertiary service with more than 15 years of MDT-based surgical management to demonstrate the outcomes and illustrative case reports that support the pivotal role of the thoracic surgeon in the MDT.

\section{Methods}

We conducted a targeted electronic search of English language publications using the following keywords:

(I) "role of thoracic surgeon";

(II) "locally advanced NSCLC";

(III) "locally advanced lung cancer";

(IV) "MDT";

(V) "multi-disciplinary team";

(VI) I AND (II OR III) AND (IV OR V).

We limited our electronic search of PubMed, Web of Science and Google Scholar to publications after 2009, then hand-searched primary source material prior to that time if referenced in relevant publications.

To provide a benchmark for MDT-based surgical management of LA-NSCLC, we queried our own databases to calculate the survival curves of groups of patients having radical lung resections for lung cancer according to the following criteria:

(I) All patients resected for LA-NSCLC, defined as any of:

(i) T stage $\geq 2 \mathrm{~b}$; OR

(ii) $\mathrm{N}$ stage $\geq 1$; OR

(iii) Procedures including:

1) Pneumonectomy;

2) Sleeve resection or pulmonary arterioplasty (i.e., resection and re-anastomosis of a major bronchus or artery to avoid a larger parenchymal resection such as pneumonectomy);

3) Superior Sulcus Resection (i.e., Pancoast tumour);

4) Extended lobectomies requiring en bloc resection of other major structures or chest wall.

(iv) Surgery after 2002 (i.e., all cases of LANSCLC discussed in MDT meetings since its inception).

(II) All patients having pre-operative radiotherapy;

(III) All patients having adjuvant radiation with or without chemotherapy;

(IV) All patients enrolled on clinical trial protocols involving surgery.

The Cox proportional hazards regression model was used 
to compare Kaplan-Meier survival curves for significant differences in univariate and multivariate analyses.

Finally, we selected illustrative cases from our databases of highly complex surgery or very LA-NSCLC to highlight the important role of the specialist thoracic surgeon in the multidisciplinary care of LA-NSCLC.

\section{Literature review}

In the recent past, there has been no conclusive evidence to confirm the positive impact of the MDT on the survival rate in patients with lung cancer (7). However, some evidence supports improved survival due to better selection of the subset of patients requiring surgical resection for LANCSLC (8).

Nevertheless, there is indirect evidence to support the benefit of specialist thoracic surgeon input to the MDT management of lung cancer. These include increasing the number of patients offered surgery and receiving complete resection, successful surgery for patients of advanced age and for patients with higher stage of cancer compared to non-specialized surgeons. Even after resecting more complex and higher stage tumors, specialist thoracic surgeons have demonstrated similar long-term outcomes historically associated with early stage tumors without increase in morbidity or mortality. Specialist thoracic surgeons are less likely to perform pneumonectomy and more likely to perform lung parenchyma-sparing surgery such as sleeve lobectomy. MDTs with specialist thoracic surgeons have been shown to have higher tissue diagnosis rates and increased use of all treatment modalities $(9,10)$.

Accurate assessment and staging of LA-NCSLC patients is of paramount importance, using minimally invasive tools for mediastinal lymph node staging, such as endobronchial ultrasound guided fine needle aspirate, mediastinoscopy and video-assisted thoracic surgery (VATS). This allows preoperative planning and discussion of multi-modality therapy where appropriate. However, despite the sensitivity of these staging tools, some patients are found to have lymph node metastasis intra-operatively ( $9 \%$ in our own series).

These patients should proceed to a complete surgical resection if the nodes are not matted or involves a single radically resectable lymph node station (11). They should also have a complete mediastinal lymph node dissection to improve their long-term survival (12). Adjuvant chemotherapy should then be strongly recommended, as this is the group with the largest relative improvement in survival (13).
A summary of NCCN guidelines on surgery for LANSCLC as they pertain to the above definition is seen in Table 1.

Enhanced recovery is an important concept in modern thoracic surgery. This entails identification of the frail or pre-frail patient, and improving their post-operative rehabilitation with high-intensity interval training (HIIT) for 2-4 weeks pre-operatively. HIIT has been shown to increase respiratory muscle strength, aerobic capacity and maximal oxygen uptake and reduce dyspnea and fatigue (14). Patients with poor pulmonary function have better surgical outcomes after HIIT, and all patients may experience less functional decline $(15,16)$. Other evidencebased enhanced recovery tools include increased patient education, minimally invasive surgery, early chest tube removal and mobilization. These should also lead to increased compliance with adjuvant chemotherapy (14). The European Society of Thoracic Surgeons have published guidelines with 45 evidence-based recommendations covering the entire peri-operative period (17). These include many multidisciplinary approaches involving dietitians, respiratory physicians, anesthesiologists, physiotherapists and nurses.

In a large retrospective study, where 504 patients with lung cancer were discussed in MDT meetings compared to 484 patients who were not, the patients discussed in MDT meetings had a significant improvement in their quality of life and increased utilization of radiotherapy, chemotherapy and palliative care. However, the survival rate did not improve (7).

In another prospective study, the impact of the MDT on the management plan for patients with lung cancer was significant, with the MDT meetings changing pre-meeting management plans in $58 \%$ of cases. In the same paper, the authors reported one third of patients planned for palliative treatment were upgraded to curative-intent management after MDT discussion. This suggests that palliative cases not presented in MDT meetings with the resulting crossspecialty discussion may not be offered the optimal management plan, especially in cases of LA-NSCLC (18).

Our conclusion from the literature is that the complexity of patient selection, multimodality therapies, surgical techniques and enhanced recovery would be extremely challenging to surmount outside of a MDT with specialist thoracic surgical membership. The incremental improvements gained over the last two decades leave little margin for error if evidence-based care is not extended to the correct populations from the outset. For those outside 
Table 1 Adapted from recommendations of the NCCN 2019 Guidelines (Version 1.2020) for resectable locally advanced NSCLC (6)

\begin{tabular}{|c|c|c|c|}
\hline Clinical presentation & Initial treatment & Adjuvant treatment & Other \\
\hline IIIA (T3N1) & Found at surgery & Chemotherapy & \\
\hline IIIA (T1-2N2) & Found at surgery & Chemotherapy & \multirow{2}{*}{$\begin{array}{l}\text { Radiotherapy or concurrent } \\
\text { chemoradiation }\end{array}$} \\
\hline IIIB (T3N2) & Found at surgery & Chemotherapy & \\
\hline $\begin{array}{l}\text { Chest wall, proximal airway, mediastinal } \\
\text { invasion (T3N0-1); stage IIIA (T4N0-1) } \\
\text { (OPTION 1) }\end{array}$ & Surgical resection* & Chemotherapy & $\mathrm{Nil}^{\star *}$ \\
\hline $\begin{array}{l}\text { Chest wall, proximal airway, mediastinal } \\
\text { invasion (T3N0-1); stage IIIA (T4N0-1) } \\
\text { (OPTION 2) }\end{array}$ & $\begin{array}{l}\text { Pre-operative concurrent } \\
\text { chemoradiation }\end{array}$ & Surgical resection* & Nil \\
\hline Stage IIIA (T1-2, T3 (other than invasive) N2) & $\begin{array}{l}\text { Induction chemotherapy } \pm \\
\text { radiotherapy }\end{array}$ & Surgical resection* & Chemotherapy \\
\hline $\begin{array}{l}\text { Separate pulmonary nodule(s), same lobe (T3, } \\
\text { N0-1); ipsilateral non-primary lobe (T4, N0-1) }\end{array}$ & Surgical resection* & Chemotherapy & $\begin{array}{l}\text { Sequential radiotherapy if } \\
\text { pN2 } \\
\text { Otherwise } \mathrm{Nil}^{\star *}\end{array}$ \\
\hline $\begin{array}{l}\text { Stage IVA (N0, M1a): contralateral lung } \\
\text { (solitary nodule vs. synchronous primary) }\end{array}$ & $\begin{array}{l}\text { Parenchyma-sparing surgical } \\
\text { resection }\end{array}$ & $\begin{array}{l}\text { Parenchyma-sparing } \\
\text { surgical resection }\end{array}$ & Chemotherapy pN1-2 \\
\hline
\end{tabular}

*, lobectomy, bilobectomy, sleeve lobectomy or pneumonectomy plus en bloc resection of invaded structures plus mediastinal lymph node dissection; ${ }^{*}$, radiotherapy or concurrent chemoradiation if positive surgical margin. NSCLC, non-small cell lung cancer; NCCN, the National Comprehensive Cancer Network.

the evidence base, outcomes depend on the full range of dedicated lung cancer and allied health specialists applying their knowledge, experience and biological principles in the patient's best interests.

\section{Surgical outcomes from 15 years of MDT-based management}

\section{$L A-N S C L C$}

Using the definition of LA-NSCLC detailed in the methods, we identified 694 patients from our database, consisting of 444 males and 250 females with a median age of 68 years (range, 21-91 years).

Only half of the resections (355/694) were considered standard lobectomy and 68 patients (10\%) received some form of neoadjuvant therapy. Pneumonectomy made up for 75 resections and there were 40 sleeve resections (including two carinal resections). It can be assumed that the latter 40 cases would have been deemed inoperable, or proceeded to pneumonectomy if treated in centers without MDT input from a specialist thoracic surgeon. These particular procedure categories carried a 30-day mortality of $4.0 \%$ and $5.3 \%$, respectively, double the $2.0 \%$ mortality of the "standard" lobectomies in the series.

More than half of the resections were attempted by minimally invasive surgery $(427 / 659 ; 65 \%)$, with 24 cases being converted to thoracotomy for failure to progress, requirement for bronchoplasty, large tumor size, or intraoperative bleeding. The 30 -day mortality was $2.5 \%$.

The overall (all-cause) 90-day mortality was 5.3\% (95\% CI: 3.6-7.0\%) and median survival was almost 5 years (Figure 1), despite $80 \%$ of cases being pathologic stage 2B or 3A (see stage spread; Figure 2). Female patients had significantly better median survival than males in univariate analysis (73 vs. 52 months; $\mathrm{P}=0.02$ ), however, this difference 


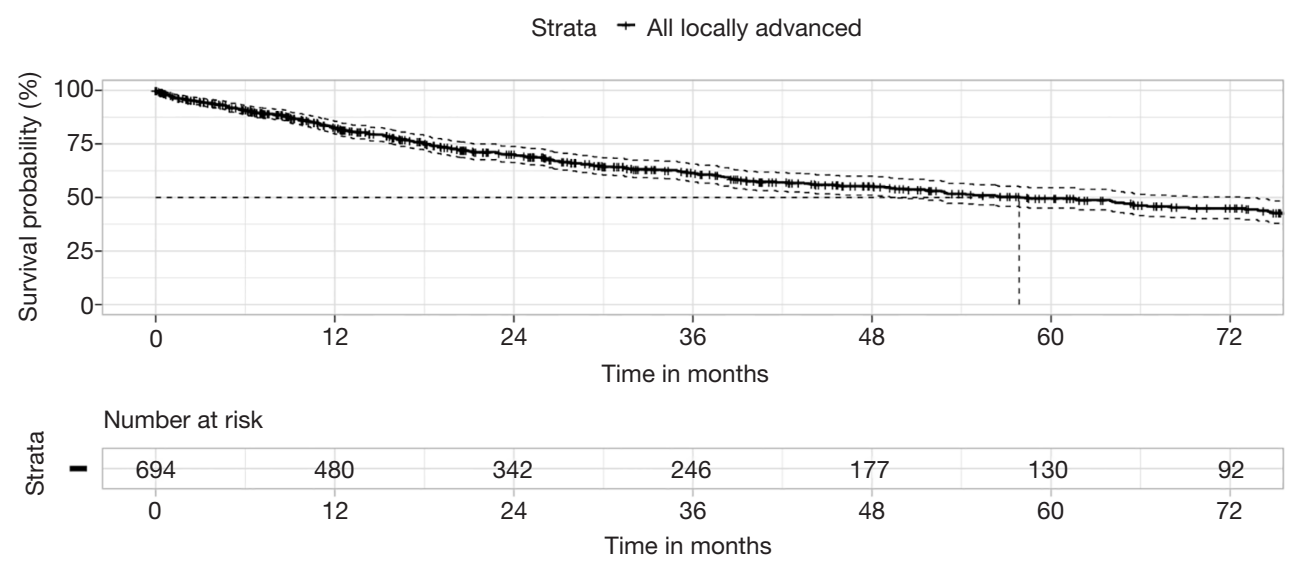

Figure 1 Kaplan-Meier survival probability curve for all patients with locally advanced NSCLC. Dashed line indicates median survival point. NSCLC, non-small cell lung cancer.

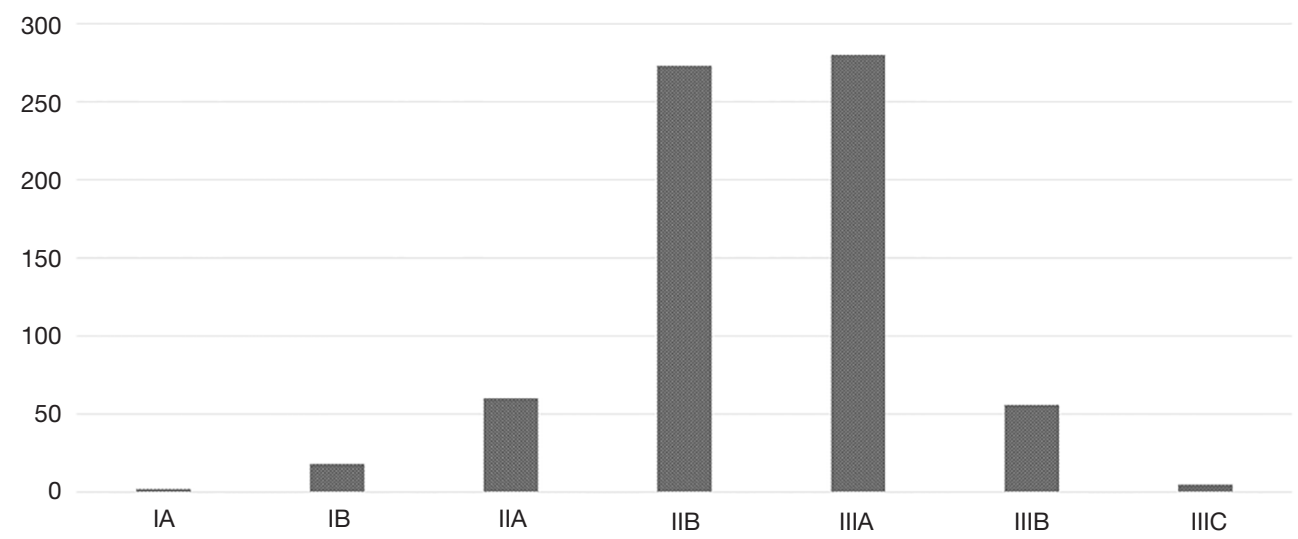

Figure 2 Stage spread (pathologic) of the pre-defined cases of locally advanced non-small cell lung cancer. Includes cases down-staged by neoadjuvant therapies.

became non-significant when controlled for age, minimally invasive surgery, histology, stage and resection margin status.

Surgery by minimally invasive techniques (VATS, robotic VATS and uniportal VATS including conversions to open surgery intra-operatively), had the most significant positive effect on long-term survival in multivariate analysis (hazard ratio $0.46, \mathrm{P}<0.0001$ ). Increasing $\mathrm{pN}$ stage (hazard ratio 1.5 , $\mathrm{P}<0.0001$ ) and involved resection margins (hazard ratio 1.7, $\mathrm{P}<0.01)$ had the greatest negative impact on survival.

\section{Pre-operative (neoadjuvant or definitive) radiotherapy then surgery}

Pre-operative radiotherapy has long been associated with higher complication rates after lung resection, with high reported mortality, especially for right pneumonectomy $(19,20)$. However, with more experience and judicious use of tissue flaps to protect the bronchial stump, there have been series reported with much lower mortality $(21,22)$.

Our own series had no mortality from pneumonectomy after radiotherapy, representing $10 / 64$ cases (16\%). However, the 30 -day and 90 -day mortality for all postradiation cases was $1.6 \%$ and $7.8 \%$, respectively (Figure 3).

Despite the early mortality, the median survival and 5 -year survival from the date of surgery was 55 months and $43 \%$, respectively. Centers without specialist thoracic surgeons willing to perform post-radiation surgery would not be able to replicate these survival results for such LA-NSCLC. 

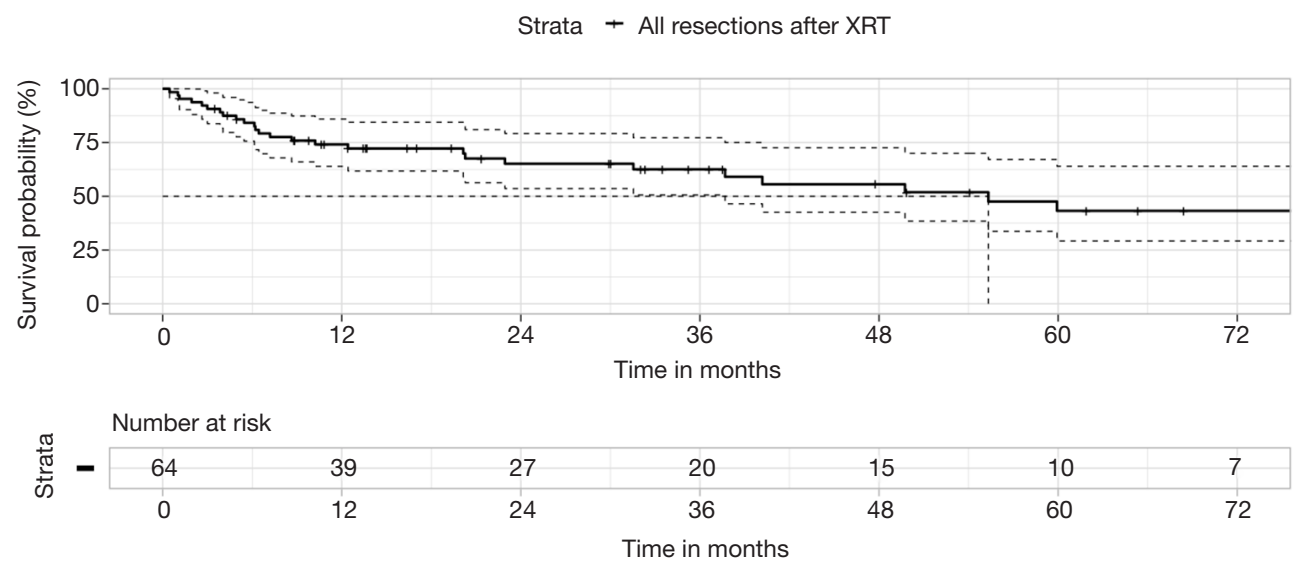

Figure 3 Kaplan-Meier survival probability curve for all patients receiving pre-operative radiotherapy (with or without chemotherapy) prior to resection of NSCLC. Dashed line indicates median survival point. XRT, radiation therapy; NSCLC, non-small cell lung cancer.

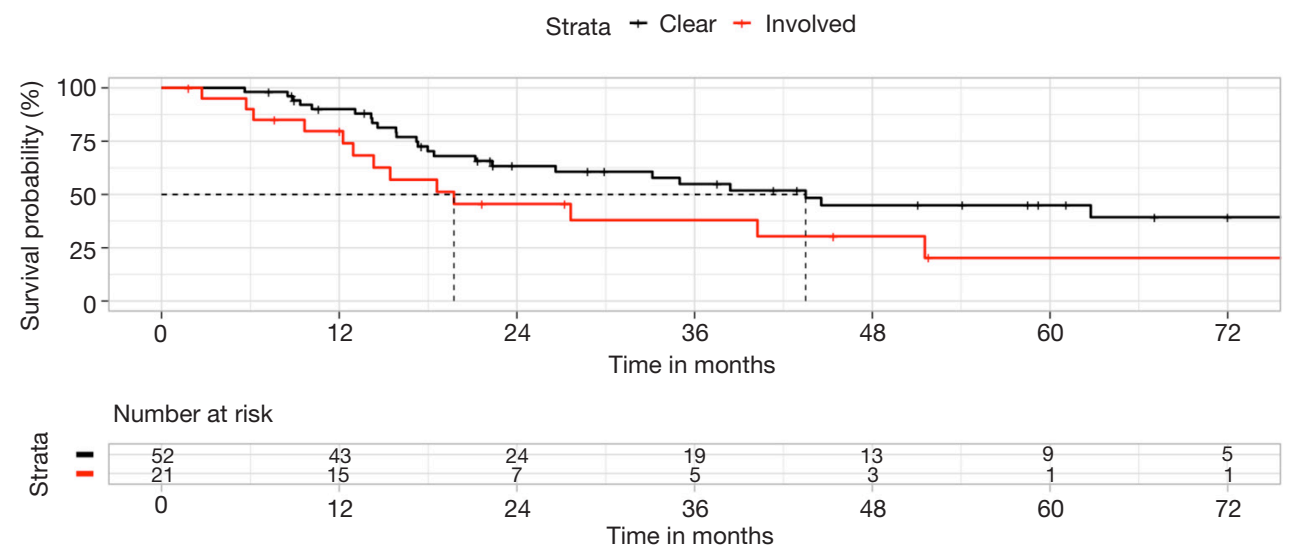

Figure 4 Kaplan-Meier survival probability curve for all patients having post-operative radiotherapy (with or without chemotherapy). Patients having radiotherapy because of surgical margins involved by tumour had significantly worse survival rates than those for adjuvant therapy of resected stage III NSCLC $(\mathrm{P}=0.04)$. Dashed line indicates median survival point. NSCLC, non-small cell lung cancer.

\section{Patients receiving post-operative radiation therapy (XRT)}

Post-operative radiotherapy is offered after discussion between the surgical and radiation oncology teams. Generally, the indications are microscopic involvement of surgical margins by tumour (R1), or less commonly macroscopic residual tumour (R2). The other indication is as adjuvant therapy for pathologic stage IIIA (N2), discovered after surgical resection. As a general policy, we recommend adjuvant radiation for either extracapsular extension or multi-station $\mathrm{N} 2$ disease in this setting. We compared the survival outcomes of patients receiving radiotherapy for positive margins against those having adjuvant radiotherapy, with or without chemotherapy.

Seventy-three patients in our database had post-operative radiotherapy, 52 for adjuvant therapy of stage IIIA disease, 19 for microscopic residual disease at surgical margins and 2 cases where macroscopic tumour remained.

There was a statistically significant difference between these groups, favoring the adjuvant setting for stage IIIA disease (Figure 4). Median survival was more than doubled compared to the residual disease group (43 vs. 20 months), as was 5 -year survival rate $(45 \%$ vs. $20 \%)(\mathrm{P}=0.04)$. This difference was in spite of the lower average stage of the residual disease cases (only 7/21 cases of $\mathrm{pN} 2$ ). 


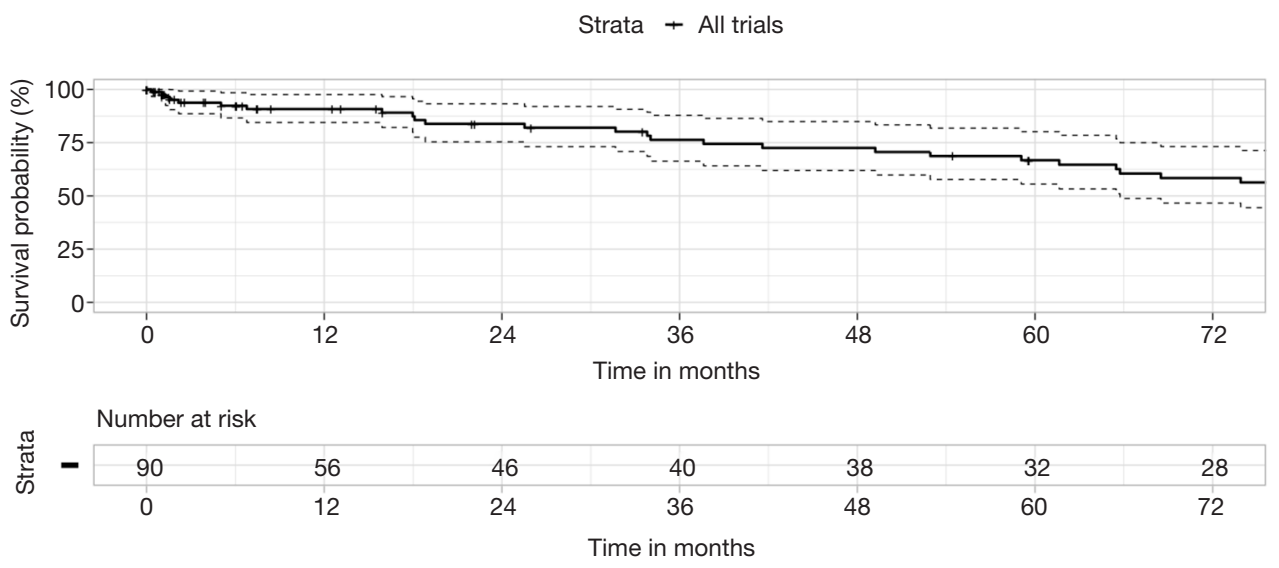

Figure 5 Kaplan-Meier survival probability curve for all patients enrolled on clinical trials. These included adjuvant chemotherapy trials, trials of sublobar resection versus lobectomy, sublobar resection versus stereotactic ablative body radiotherapy and trials of radical lymphadenectomy versus systematic lymph node sampling.

\section{Clinical trial participants}

A feature of MDT is access to, and promotion of clinical research. An MDT with specialist thoracic surgical support can support a wider range of trials, beyond the standard sponsored drug A versus drug B trial in the metastatic setting. Additionally, clinical trial-based surgery allows for extended and detailed follow-up. Trials included ACOSOG Z30, CALGB 30506 and 140503, MAGRIT, RADIANT, BR31, ALINA, StableMates, Impower 030 and our own investigator-initiated surgical trials. We studied the surgical outcomes of 90 patients enrolled on registered clinical trials and found that they had a low 30-day mortality of $1.1 \%$ and an excellent median survival of 10 years. The 5 -year overall survival was $67 \%$ (Figure 5 ).

\section{Audit of outcome in MDT-based surgical management of LA-NSCLC}

Audit of outcome should be a routine part of MDT activity for the surgical team, extending beyond the blunt tool of post-operative mortality. To justify operating on LANSCLC, the thoracic surgical oncologist must benchmark the measure of response to surgery-a tumor-free surgical margin. We believe that the rate of microscopic surgical margin involvement should be less than $5 \%$ for all comers. This rises with the percentage of locally advanced cases, but should be maintained well below the $10 \%$ mark. We studied the results of the defined locally advanced cases and found that over $94 \%$ had clear margins (617/659 cases).
Of the involved margins only 3 cases out of 42 were due to macroscopic residual tumour.

Resource management and harm minimization are also important in surgical oncology. One of the surrogates for cost and morbidity is the post-operative length of stay. We calculated our median length of post-operative hospital stay as 7 days, with interquartile range of 5-11 days. This compares well to historical median length of stay for all lung resections.

In conclusion, our results from "real world" MDT-based specialist thoracic surgical care of patients with LA-NSCLC confirm that excellent long-term outcomes can be achieved with low short term mortality, acceptable morbidity and resource utilization.

\section{Case reports}

We chose four illustrative cases to highlight the value of specialist thoracic surgeons in the multidisciplinary care of the patient with LA-NSCLC. These cases were all considered inoperable by general surgeons, or generalist cardiothoracic surgeons without special interest in surgical oncology, before being referred to our central MDT for review. They highlight that assessment of physiological age, rather than chronological age, is part of the art of thoracic surgery, as is determining how far to extend complex resection without major detriment to quality of life (or survival). Our assessment of physiological age includes functional and social status, self-reported health status and additional tests including 6-minute 
Table 2 Typical illustrative cases where specialist thoracic surgical input was critical for optimal patient outcome in locally advanced NSCLC

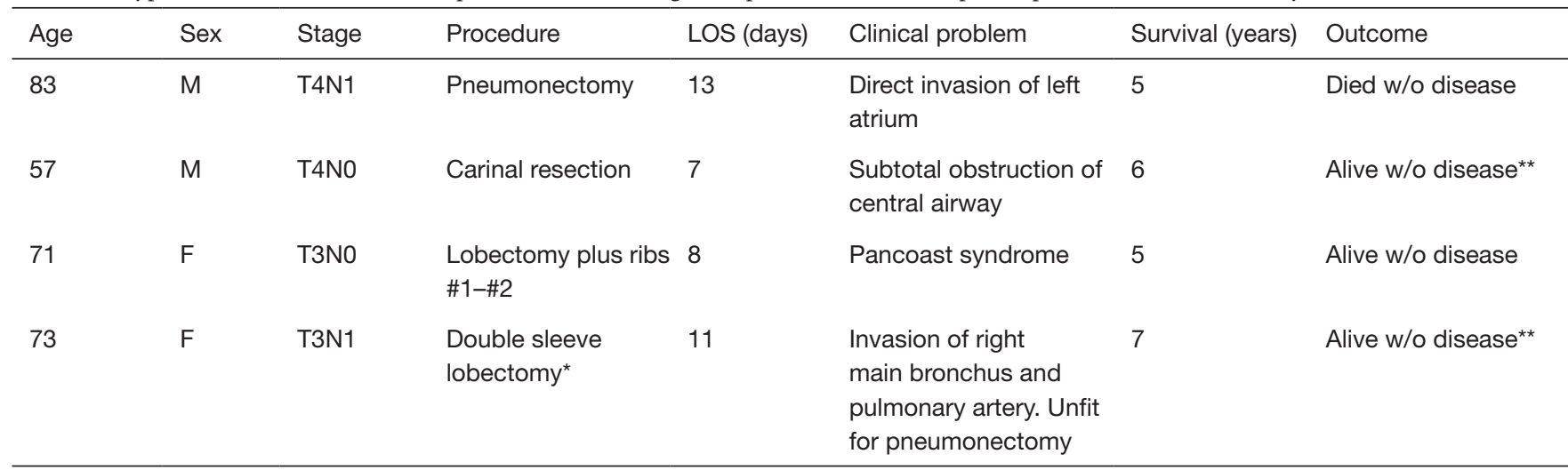

${ }^{*}$, resection and reconstruction of pulmonary artery wall and main bronchus; ${ }^{* *}$, later developed metachronous primary lung cancers, treated with radical radiotherapy. NSCLC, non-small cell lung cancer.

walk test, cardiopulmonary exercise testing, stress echocardiography, thallium stress tests and quantitative V/ Q scans. Reconstructive and parenchyma-sparing options are also more likely to be offered in centralized high volume thoracic surgical centers, which are linked to MDTs. Our team consider pneumonectomy a procedure of last choice if a reconstruction is not possible, even though it is often the easier technical procedure.

Table 2 summarizes the illustrative case reports detailed below.

\section{The elderly (fit) patient}

An 83-year-old retired male from regional Australia presented with cough and increasing shortness of breath and was found to have a large left central squamous lung cancer invading his left atrium. A pneumonectomy was performed with en bloc resection of a cuff of left atrium. Clear surgical margins were achieved and the patient discharged home after 13 days with a final pathologic stage of T4N1M0. He lived at home for 4 years, then was admitted to a low care nursing facility for reasons unrelated to his cancer. He died without evidence of lung cancer 5 years after resection.

\section{The central airway tumor}

A 57-year-old farmer from rural Australia presented with increasing stridor and shortness of breath. A CT scan revealed a $2-\mathrm{cm}$ rounded intraluminal lesion at the tracheo-bronchial bifurcation. He was borderline fitness for pneumonectomy based on pulmonary function tests. A laser bronchoscopy was preformed urgently to re-canalize his airway and a diagnosis of squamous lung cancer was made. He underwent right thoracotomy and carinal resection without loss of pulmonary parenchyma. Clear surgical margins were achieved and the patient discharged home after 7 days with a final pathologic stage of T4N0M0. The following year an episode of hemoptysis led to discovery of a similar lesion in the left main bronchus, $2 \mathrm{~cm}$ from the previous anastomosis. It was unclear whether this was a local recurrence, or a new metachronous squamous lung cancer. After repeat laser bronchoscopy, he had radical combined chemotherapy and radiotherapy (60 Gy) and remained disease-free after 5 years of follow-up.

\section{The superior sulcus tumor (Pancoast)}

A 71-year-old female presented with posterior chest pain radiating into axilla was referred after the finding of a right apical mass with destruction of cortex of first and second ribs. A two-surgeon approach was used to perform VATS right upper lobectomy from an anterior approach, and a separate minimally invasive paraspinal incision to divide the involved ribs. A re-resection of the $2^{\text {nd }}$ rib stump was required 4 weeks later for suspected involved rib margin, however there was no residual tumour found. Her admission time was 8 days for both procedures. The final pathologic stage was T3N1M0. She remained disease-free after more than 5 years of follow-up.

\section{The major broncho-vascular reconstruction}

A 73-year-old female with emphysema and atrial fibrillation was referred with increasing shortness of breath, initially 
thought to be an exacerbation of her chronic airway disease. A central tumour was identified on CT scan which occluded the right upper lobe and appeared to invade the main pulmonary artery. Bronchoscopy confirmed a diagnosis of squamous lung cancer. She underwent a sleeve resection of the right upper lobe and right main bronchus with reimplantation of the bronchus intermedius. A resection of the involved wall of the right pulmonary artery was reconstructed with autologous pericardium. The patient was discharged home after 11 days with a final pathologic stage of T3N1M0. She developed a new lung adenocarcinoma 6 years later, which was treated with radiotherapy. She remains disease-free after 7 years of follow-up.

\section{Conclusions}

To optimize patient outcomes, particularly for LA-NSCLC, lung cancer MDT meetings should be organized to include specialist thoracic surgeons as members of the team. Smaller centers without specialist thoracic surgical services should refer all patients with LA-NSCLC to a centralized MDT for a surgical opinion and should never assume a patient with LA-NSCLC is inoperable without this specialized thoracic surgical and MDT review.

\section{Acknowledgments}

The senior author wishes to thank his thoracic surgical colleagues, Dr. Naveed Alam and Dr. Stephen Barnett, for allowing their data to be included in the audit of surgical outcomes. The authors also wish to acknowledge the $15+$ years of clinical contribution of their MDT colleagues across the Victorian Comprehensive Cancer Centre alliance, including St Vincent's Hospital (Melbourne), Peter MacCallum Cancer Centre and the Royal Melbourne Hospital.

Funding: None.

\section{Footnote}

Provenance and Peer Review: This article was commissioned by the Guest Editor (Emily Stone) for the series "Lung Cancer Multidisciplinary Care" published in Translational Lung Cancer Research. The article was sent for external peer review organized by the Guest Editor and the editorial office.

Conflicts of Interest: Both authors have completed the
ICMJE uniform disclosure form (available at http://dx.doi. org/10.21037/tlcr.2019.11.22). The series "Lung Cancer Multidisciplinary Care" was commissioned by the editorial office without any funding or sponsorship. The authors have no other conflicts of interest to declare.

Ethical Statement: The authors are accountable for all aspects of the work in ensuring that questions related to the accuracy or integrity of any part of the work are appropriately investigated and resolved. The multi-site quality audit of surgical outcomes was approved under St. Vincent's HREC: 030/12, LNR HREC reference number: HREC/17/SVHM/82 and LRR 205/17, covering the Victorian Comprehensive Cancer Centre alliance of hospitals.

Open Access Statement: This is an Open Access article distributed in accordance with the Creative Commons Attribution-NonCommercial-NoDerivs 4.0 International License (CC BY-NC-ND 4.0), which permits the noncommercial replication and distribution of the article with the strict proviso that no changes or edits are made and the original work is properly cited (including links to both the formal publication through the relevant DOI and the license). See: https://creativecommons.org/licenses/by-nc-nd/4.0/.

\section{References}

1. Ellis PM. The importance of multidisciplinary team management of patients with non-small-cell lung cancer. Curr Oncol 2012;19:S7-15.

2. Samson P, Patel A, Crabtree TD, et al. Multidisciplinary Treatment for Stage IIIA Non-Small Cell Lung Cancer: Does Institution Type Matter? Ann Thorac Surg 2015;100:1773-9.

3. Dickhoff C, Hartemink KJ, van de Ven PM, et al. Trimodality therapy for stage IIIA non-small cell lung cancer: benchmarking multi-disciplinary team decisionmaking and function. Lung Cancer 2014;85:218-23.

4. Powell HA, Baldwin DR. Multidisciplinary team management in thoracic oncology: more than just a concept? Eur Respir J 2014;43:1776-86.

5. Pan CC, Kung PT, Wang YH, et al. Effects of multidisciplinary team care on the survival of patients with different stages of non-small cell lung cancer: a national cohort study. PLoS One 2015;10:e0126547.

6. Ettinger DS, Wood DE, Aggarwal C, et al. NCCN Clinical Practice Guidelines in Oncology: Non-Small Cell 
Lung Cancer. Version 1.2020, November 2019: NSCL1NSCL31. Available online: https://www.nccn.org/ professionals/physician_gls/pdf/nscl.pdf

7. Boxer MM, Vinod SK, Shafiq J, et al. Do multidisciplinary team meetings make a difference in the management of lung cancer? Cancer 2011;117:5112-20.

8. Hanagiri T, Takenaka M, Oka S, et al. Results of a surgical resection for patients with stage IV non-small-cell lung cancer. Clin Lung Cancer 2012;13:220-4.

9. Martin-Ucar AE, Waller DA, Atkins JL, et al. The beneficial effects of specialist thoracic surgery on the resection rate for non-small-cell lung cancer. Lung Cancer 2004;46:227-32

10. Wright $\mathrm{G}$. The benefits of appointing a specialist thoracic surgeon to a lung cancer service. Lung Cancer 2005;47:425-6.

11. Varela G, Thomas PA. Surgical management of advanced non-small cell lung cancer. J Thorac Dis 2014;6 Suppl 2:S217-23.

12. Mokhles S, Macbeth F, Treasure T, et al. Systematic lymphadenectomy versus sampling of ipsilateral mediastinal lymph-nodes during lobectomy for nonsmall-cell lung cancer: a systematic review of randomized trials and a meta-analysis. Eur J Cardiothorac Surg 2017;51:1149-56.

13. Pignon JP, Tribodet H, Scagliotti GV, et al. Lung adjuvant cisplatin evaluation: a pooled analysis by the LACE Collaborative Group. J Clin Oncol 2008;26:3552-9.

14. Osarogiagbon RU, Veronesi G, Fang W, et al. Early-Stage NSCLC: Advances in Thoracic Oncology 2018. J Thorac Oncol 2019;14:968-78.

15. Licker M, Karenovics W, Diaper J, et al. Short-Term Preoperative High-Intensity Interval Training in Patients
Awaiting Lung Cancer Surgery: A Randomized Controlled Trial. J Thorac Oncol 2017;12:323-33.

16. Sebio García R, Yáñez-Brage MI, Giménez Moolhuyzen $\mathrm{E}$, et al. Preoperative exercise training prevents functional decline after lung resection surgery: a randomized, singleblind controlled trial. Clin Rehabil 2017;31:1057-67.

17. Batchelor TJP, Rasburn NJ, Abdelnour-Berchtold E, et al. Guidelines for enhanced recovery after lung surgery: recommendations of the Enhanced Recovery After Surgery (ERAS®) Society and the European Society of Thoracic Surgeons (ESTS). Eur J Cardiothorac Surg 2019;55:91-115.

18. Ung KA, Campbell BA, Duplan D, et al. Impact of the lung oncology multidisciplinary team meetings on the management of patients with cancer. Asia Pac J Clin Oncol 2016;12:e298-304.

19. Martin J, Ginsberg RJ, Abolhoda A, et al. Morbidity and mortality after neoadjuvant therapy for lung cancer: the risks of right pneumonectomy. Ann Thorac Surg 2001;72:1149-54

20. Daly BDT, Fernando HC, Ketchedjian A, et al. Pneumonectomy after high-dose radiation and concurrent chemotherapy for nonsmall cell lung cancer. Ann Thorac Surg 2006;82:227-31.

21. Sonett JR, Suntharalingam M, Edelman MJ, et al. Pulmonary resection after curative intent radiotherapy (>59 Gy) and concurrent chemotherapy in non-small-cell lung cancer. Ann Thorac Surg 2004;78:1200-5.

22. Gudbjartsson T, Gyllstedt E, Pikwer A, et al. Early surgical results after pneumonectomy for non-small cell lung cancer are not affected by preoperative radiotherapy and chemotherapy. Ann Thorac Surg 2008;86:376-82.
Cite this article as: $\mathrm{Al}$ Zaidi M, Wright GM. Locally advanced non-small cell lung cancer: the place of specialist thoracic surgery in the multidisciplinary team. Transl Lung Cancer Res 2020;9(4):1680-1689. doi: 10.21037/tlcr.2019.11.22 